\title{
Utilisation Des Indices De Qualité Et De Pollution Organique Dans L'évaluation De La Qualité Physico- Chimique Des Eaux Superficielles Des Oueds Moulouya et Ansegmir (Haute Moulouya, NE Du Maroc)
}

\author{
My Hachem Bekri, Doctorant \\ Abdellah El Hmaidi, Professeur \\ Hajar Jaddi, Doctorante \\ Habiba Ousmana, Docteur
}

Equipe Sciences de l'Eau et Ingénierie de l'Environnement, Faculté des Sciences, Université Moulay Ismaïl, Zitoune, Meknès, Maroc.

\section{Zahra Kasse, Docteur}

Lycée Anassi (Annexe), Direction Régionale, Meknès, Ministère de

l'Education Nationale, Maroc.

\section{El Mati El Faleh, Professeur}

Equipe Géosciences, Patrimoine et Substances Utiles (GPS), Faculté des

Sciences, Université Moulay Ismail, Zitoune, Meknès, Maroc.

\section{Ali Essahlaoui, Professeur \\ Abdelhadi El Ouali, Professeur}

Equipe Sciences de l'Eau et Ingénierie de l'Environnement, Faculté des

Sciences, Université Moulay Ismaïl, Zitoune, Meknès, Maroc.

\section{Doi:10.19044/esj.2020.v16n27p55 URL:http://dx.doi.org/10.19044/esj.2020.v16n27p55}

\section{Résumé}

Les eaux superficielles sont soumises à de fortes pressions anthropiques suscitées par le développement et l'extension des activités agricoles et également par les activités industrielles et domestiques. La pollution est un grand problème pour l'environnement en raison des rejets déversés dans les rivières et de l'utilisation excessive des fertilisants agricoles et des rejets d'origine urbaine et industrielle. L'objectif de cette étude est d'évaluer la qualité et l'état de la pollution organique des eaux superficielles la Haut Moulouya en se basant sur l'indice de qualité de l'eau (IQE) et l'indice de pollution organique (IPO) durant trois campagnes de prélèvement effectuées 
entre les mois de mars et août de l'année 2014. Dix stations ont été étudiées le long des oueds Moulouya et Ansegmir jusqu'à l'amont du barrage Hassan II. Les résultats sont visualisés par l'utilisation des SIG via la réalisation de cartes thématiques. Ils ont révélé que la majorité des stations indiquent une excellente qualité d'eau et ne présentent aucune pollution organique à l'exception des stations de Boumia (M5) et de Zaida (M6) qui présentent respectivement des eaux de mauvaise qualité et une faible pollution au niveau de Boumia et des eaux non potables avec forte pollution organique au niveau de Zaida. Cette dégradation environnementale anthropique enregistrée en aval des deux communes urbaines proviendrait de l'utilisation d'engrais agricoles azotés et phosphatés et surtout des rejets d'eaux usées domestiques et industrielles non traitées de ces deux communes. La variation saisonnière reste discrète et traduit une légère tendance à la dégradation des eaux de la Haute Moulouya pendant les basses eaux de la période estivale.

Mots clés: Haute Moulouya, Eaux superficielles, Physico-chimie, IQE, Indice de qualité d'eau IPO, Indice de pollution organique, Rejets urbains, Variation saisonnière 


\title{
Use of Quality and Organic Pollution Indices in the Assessment of the Physico-Chemical Quality of Surface Waters in the Moulouya and Ansegmir Rivers (Uppeer Moulouya, NE of Morocco)
}

\author{
My Hachem Bekri, Doctorant \\ Abdellah El Hmaidi, Professeur \\ Hajar Jaddi, Doctorante \\ Habiba Ousmana, Docteur
}

Equipe Sciences de l'Eau et Ingénierie de l'Environnement, Faculté des Sciences, Université Moulay Ismaïl, Zitoune, Meknès, Maroc.

\section{Zahra Kasse, Docteur}

Lycée Anassi (Annexe), Direction Régionale, Meknès, Ministère de

l'Education Nationale, Maroc.

El Mati El Faleh, Professeur

Equipe Géosciences, Patrimoine et Substances Utiles (GPS), Faculté des

Sciences, Université Moulay Ismail, Zitoune, Meknès, Maroc.

\section{Ali Essahlaoui, Professeur \\ Abdelhadi El Ouali, Professeur}

Equipe Sciences de l'Eau et Ingénierie de l'Environnement, Faculté des

Sciences, Université Moulay Ismaïl, Zitoune, Meknès, Maroc.

Abstract

Surface water is subject to strong anthropogenic pressures caused by the development and extension of agricultural activities and also by industrial and domestic activities. Pollution is a serious problem for the environment due to discharges dumped into rivers and excessive use of agricultural fertilizers and discharges from urban and industrial sources. The objective of this study is to assess the quality and state of organic pollution in surface water in Upper Moulouya based on the water quality index (WQI) and the organic pollution index (IPO) during three sampling campaigns carried out between March and August of 2014. Ten stations were studied along the Moulouya and Ansegmir wadis up to the Hassan II dam. The results are visualized through the use of GIS through the production of thematic maps. They revealed that the majority of stations indicate excellent water quality and show no organic pollution with the exception of Boumia (M5) and Zaida (M6) stations which respectively have poor quality water and low pollution. at Boumia and non-drinking water 
with strong organic pollution at Zaida. This anthropogenic environmental degradation recorded downstream of the two urban communes would come from the use of nitrogen and phosphate agricultural fertilizers and above all from the discharge of untreated domestic and industrial wastewater from these two communes. The seasonal variation remains discreet and reflects a slight tendency towards degradation of the waters of the Upper Moulouya during the low waters of the summer period.

Keywords: Upper Moulouya, Surface water, Physico-chemistry, WQI, Water Quality Index, OPI, Organic Pollution Index, Urban discharges, Seasonal variation

\section{Introduction}

Riche en milieux aquatiques, le Maroc est parmi les pays d'Afrique les plus menacés par le fléau de la pollution de ses eaux (Khamar et al., 2000 ; Mutin, 2000 ; Azzaoui et al., 2002 ; Taybi et al., 2016).

L'eau est une ressource naturelle indispensable à la vie dans tout écosystème (Tampo et al., 2015). La connaissance de sa qualité donne une vision globale des risques afin d'assurer la protection des ressources et pour déterminer les sources possibles d'altération de la qualité de l'eau (Myers., 2015), (Normatov et al.,2015).

Les sources d'eau peuvent être principalement sous forme de rivières, de lacs, d'eau de pluie, d'eau souterraine, etc. En plus des besoins en alimentation en eau potable, les ressources en eau jouent un rôle vital dans divers secteurs de l'économie du Maroc tels que l'agriculture, la foresterie, les activités industrielles, l'élevage, la production d'hydroélectricité, etc. La disponibilité et la qualité de l'eau en surface ou souterraine, se sont détériorées en raison de certains facteurs importants comme la croissance démographique, la pollution causée par l'industrialisation ainsi que l'urbanisation, etc.

La qualité de l'eau est un critère important pour faire répondre à la demande et à l'approvisionnement en eau. Garantir une qualité d'eau douce adaptée aux besoins humains et écologiques est donc un aspect important de la gestion intégrée de l'environnement et du développement durable. Pour représenter de manière claire la qualité de l'eau, différents indices de la qualité de l'eau sont utilisés pour évaluer la qualité des eaux superficielles.

L'indice de la qualité globale de l'eau IQE et de pollution étaient des outils très utiles pour prendre la bonne décision et évaluer de façon comparative la qualité de l'eau et le degré de pollution dans le temps et dans l'espace à l'échelle du bassin versant (Talhaoui et al., 2020 ; El Hmaidi et al., 2020).

Le présent travail est basé sur l'utilisation des indices de qualité globale de l'eau (IQE) et de pollution organique (IPO) en fonction des paramètres physico-chimiques des eaux superficielles du bassin versant de la Haute 
Moulouya. L'objectif est d'évaluer la qualité et l'état de pollution organique des eaux superficielles au niveau de dix stations situées en amont du barrage Hassan II (Haute Moulouya, NE, Maroc) pendant trois compagnes de l'année 2014. Les cartes thématiques finales de distribution de l'IQE et l'IPO ont été préparées à l'aide du système d'information géographique SIG.

\section{Présentation de la zone d'étude}

L'Oued Moulouya est l'un des plus grands fleuves du Maroc. Il prend ses sources à Alemssid (Région d'Aghbala) à $2000 \mathrm{~m}$ d'altitude près de Midelt à la jonction de la chaîne Moyenne et Haute Atlasique. Son écoulement s'étend sur plus de $500 \mathrm{~km}$ avec une forte variabilité. Il fournit de l'eau potable aux centres urbains, il est largement utilisé pour la consommation domestique, l'irrigation et même pour l'abreuvement du bétail (El Hachimi et al., 2005).

La zone d'étude est située dans la Haute Moulouya. Elle forme une cuvette réservant du matériel sédimentaire issu du Haut et du Moyen Atlas. Elle est limitée au Sud par le Haut Atlas, au Nord et Nord-Est par le Moyen Atlas. Sa superficie couvre près de $4500 \mathrm{Km}^{2}$, dont $85 \%$ de plaines aux alentours de $2000 \mathrm{~m}$ et $15 \%$ de montagnes culminant à Jbel El Ayachi avec $3750 \mathrm{~m}$ d'altitude (Fig.1). Le district minier de la Haute Moulouya (Zaïda, Mibladen, et Aouli) est l'un des plus gros districts $\mathrm{Pb}-\mathrm{Zn}$ au royaume du Maroc.
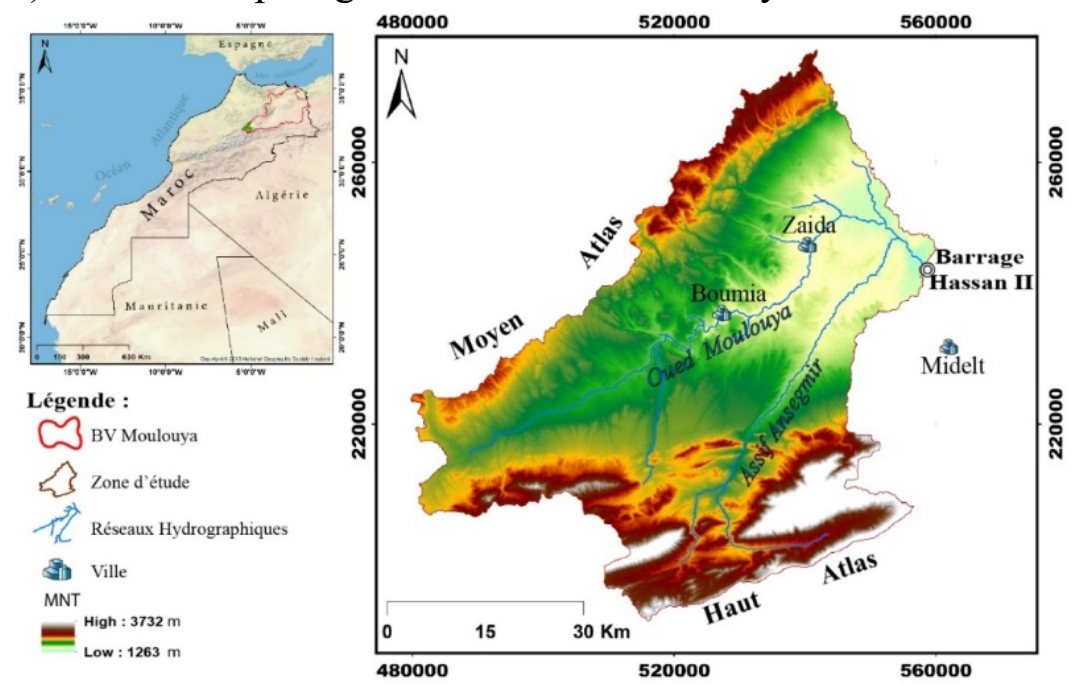

Figure 2. Situation géographique du bassin versant de la Haute Moulouya.

Le climat de la Haute Moulouya est largement influencé par l'orographie atlasique. L'été est très chaud et orageux $\left(33,8^{\circ} \mathrm{C}\right.$ en juillet), l'hiver est froid $\left(-1,3^{\circ} \mathrm{C}\right.$ en janvier) et la température moyenne annuelle est de $13^{\circ} \mathrm{C}$. Le climat est doté d'une atmosphère continentale sèche relativement fraîche lorsque les vents d'ouest dominent (Combe \& Simonot, 1971). La pluviométrie moyenne annuelle est d'environ $300 \mathrm{~mm}$. Elle varie entre $210 \mathrm{~mm}$ en aval (station 
Ansegmir) et s'élève jusqu'à $379 \mathrm{~mm}$ en escaladant les frontières montagneuses (station Oufounes), avec occasionnellement de gros éclats de nuages et la zone est parfois soumise à des chutes de neige survenant entre novembre et février.

Le couvert végétal, dispersé, est représenté essentiellement par des touffes de l'alfa (Stipa tenacissima L.) et d'artémisiaes à armoise blanche (Artemisia herba alba), qui subissent l'action d'un surpâturage continu et prolongé (Rhanem, 2009).

Le bassin versant de la Haute Moulouya est drainé par deux principaux tributaires : oued Moulouya et oued Ansegmir qui reçoivent tout au long de leurs cours amont les rejets domestiques des agglomérations (Boumia, Zaida, Itzer et Tounfite) (ABHM, 2009) ainsi que les déchets résultant de l'agriculture développée sur les vallées des deux oueds. En plus, l'oued Moulouya draine les eaux émanant du district minier abandonné de Zaida.

Les débits moyens des deux oueds au cours de l'année 2011-2012 montrent que le régime hydrologique de la Moulouya évoluait à peu près de la même manière que celui de l'oued Ansegmir (Fig.2). Cependant, lors du mois de novembre, le débit moyen d'Ansegmir était plus important que celui de Moulouya (Chahboune et al., 2014).

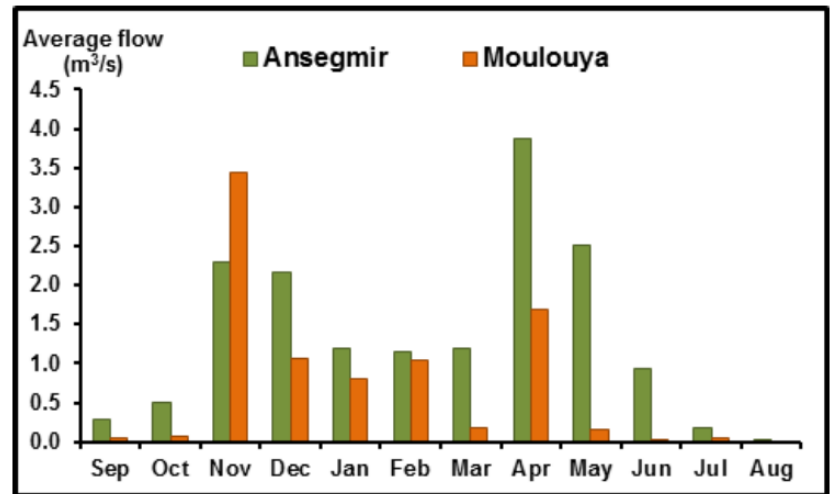

Figure 2. Évolution du débit moyen mensuel au niveau des stations hydrologiques de Zaida et Ansegmir pendant l'année 2011-2012 (Chahboune et al., 2014).

D'un point de vue géologique (Fig.3), différentes formations affleurent au niveau du bassin versant de la Haute Moulouya dont l'âge s'étend du Paléozoïque, représentant le socle, au Quaternaire (Combe \& Simonot, 1971 ; Ahamrouni, 1996). Ainsi, le Paléozoïque est représenté essentiellement par des schistes intrusés par des granitoïdes. Les granites forment des massifs primaires de Boumia et d'Ahouli. Le Trias affleure en discordance angulaire sur le Paléozoïque. Il est formé par des formations argilo-salines détritiques rouges et des basaltes très altérés. Le Jurassique est représenté par des faciès calcaro-dolomitiques très karstifiés et des dépôts terrigènes rouges formant le paléo seuil de la haute Moulouya. Le Crétacé (Cénomanien) commence par 
des conglomérats à ciment calcaire ou gréseux, grès, argiles et marnes. Il se poursuit avec des calcaires turoniens en barres intercalées par des lits d'argile sur lesquels viennent se déposer des séries évaporitiques surtout gypseuses (Nasloubi, 1993). Le Tertiaire est représenté par des dépôts paléogènes continentaux (conglomérats, grès et marnes, et dolomies) suivis de conglomérats argileux, calcaires lacustres, marnes et gypse du Néogène (Naji, 2004).

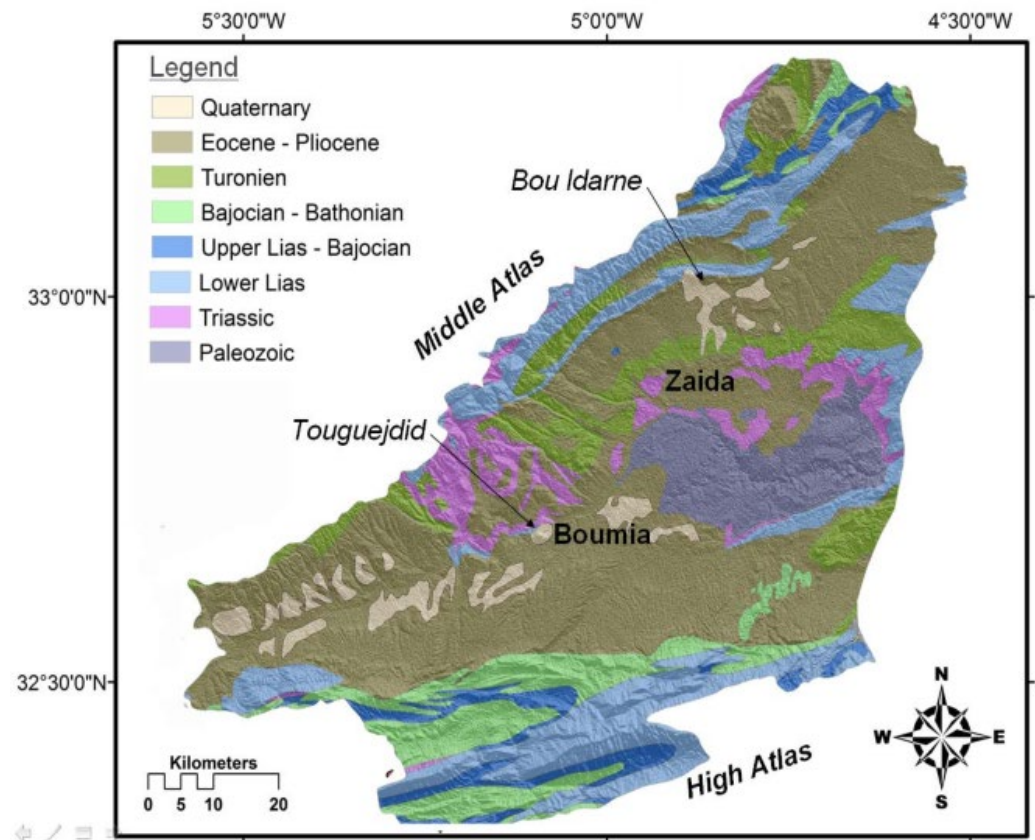

Figure 3. Carte géologique du bassin de la Haute Moulouya. D'après la carte géologique du Maroc au 1/1000000 (Diani et al., 2017).

Le Quaternaire est constitué par des formations les plus récentes qui viennent se déposer au-dessus de toutes les autres formations précédentes. Ce sont des terrasses fluviatiles qui affleurent sous forme de séries emboitées et recouvertes de dépôts essentiellement limoneux et conglomératiques (Amrani, 2007 ; Laabidi et al., 2014).

\section{Méthodologie et données utilisées}

\section{Base de données}

Dans le cadre de cette étude, les données utilisées comprennent les données relatives aux analyses physico-chimiques des eaux superficielles des oueds Moulouya et Ansegmir situés dans la Haute Moulouya (Taybi et al., 2016). L'échantillonnage a été effectué durant trois campagnes étalées sur six mois successivement pendant les mois de mars-avril, mai-juin et juillet-août 2014 (Fig. 4). Les stations d'échantillonnage ont été choisies en fonction des différentes activités identifiées dans la zone d'étude (eaux usées domestiques, 
industrielles et agricoles). Ainsi, 30 échantillons d'eau ont été prélevés dans 10 stations réparties le long des rivières Moulouya et Ansegmir (Fig. 4).

Plusieurs stations ont été choisies en amont du bassin versant à proximité des sources de la rivière Moulouya pour les stations $\mathrm{S} 1, \mathrm{~S} 2$ et $\mathrm{S} 3$ et de la rivière Ansegmir pour les stations S7, S8 et S9 un peu dans la zone de jonction du Haut et du Moyen Atlas. Elles représentent des stations de références loin des apports anthropiques domestiques, industriel et agricole. Les stations S4 et S5 se situent respectivement en amont et en aval de la commune urbaine de Boumia. La station S6 se trouve dans la commune de Zaida et proche de son centre minier $\mathrm{Pb}-\mathrm{Zn}$. En fin, la station $\mathrm{S} 10$ est située sur la rivière Ansegmir près des surfaces agricoles et arboricoles juste avant le barrage Hassan II.

Les paramètres physico-chimiques comprennent le $\mathrm{pH}$, l'oxygène dissous $\mathrm{OD}$, la conductivité électrique $\mathrm{CE}$ et la température $\mathrm{T}^{\circ} \mathrm{C}$, mesurés (in situ) sur le terrain, et les sulfates $\left(\mathrm{SO}_{4}{ }^{2-}\right)$, la demande biologique en oxygène après 5 jours $\left(\mathrm{DBO}_{5}\right)$, les Orthophosphates $\left(\mathrm{PO}_{4}{ }^{3-}\right)$, l'Ammonium $\left(\mathrm{mg} \mathrm{N}-\mathrm{NH}_{4}\right)$ et les Nitrates (mg N-NO${ }^{-}$) analysés au laboratoire (Taybi et al., 2016). Ces paramètres ont été déterminés selon les méthodes d'analyses préconisées par les normes AFNOR (1997) et par Rodier et al. (2009).

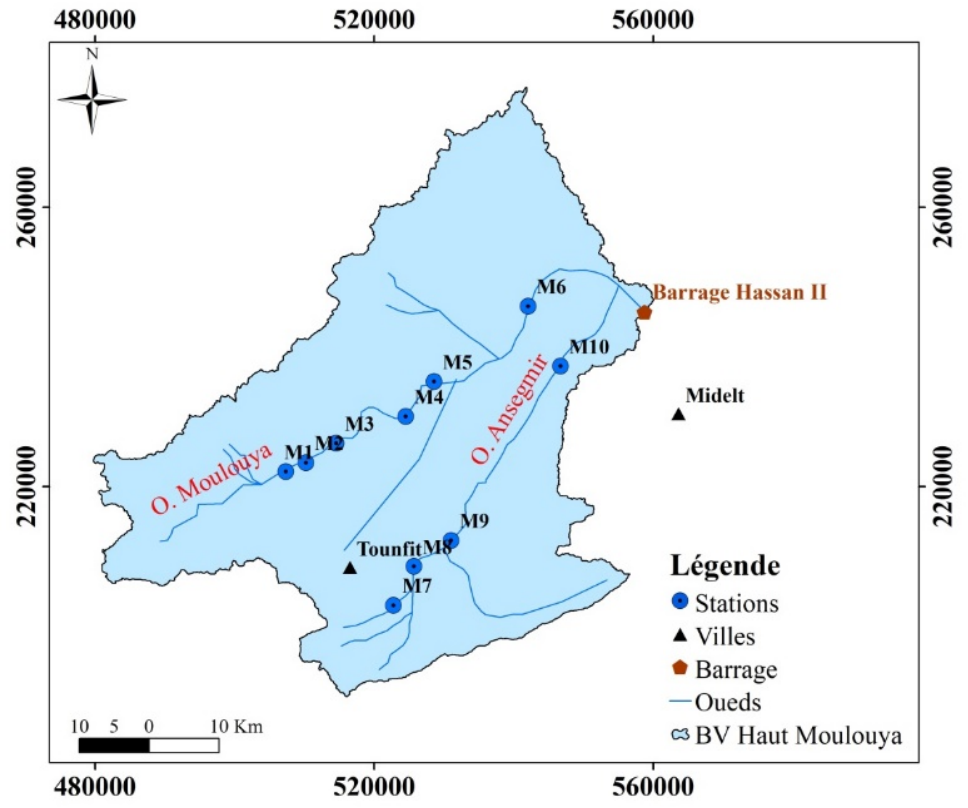

Figure 4. Localisation des stations d'étude du bassin versant la Haute Moulouya en amont du barrage Hassan II.

L'évaluation et la visualisation des résultats ont été effectuées à l'aide du logiciel Excel 2010, RStudio et des logiciels destinés au système d'information géographique SIG. 


\section{Calcul de l'Indice de qualité de l'eau (IQE)}

Neuf paramètres importants $\left(\mathrm{pH}, \mathrm{OD}, \mathrm{CE}, \mathrm{T}^{\circ} \mathrm{C}, \mathrm{SO}_{4}{ }^{2-}, \mathrm{DBO}_{5}, \mathrm{PO}_{4}{ }^{3-}, \mathrm{N}-\right.$ $\mathrm{NH}_{4}$ et $\mathrm{N}-\mathrm{NO}_{3}$ ) ont été sélectionnés pour calculer l'Indice de qualité de l'eau IQE. Cet indice est une technique de classification de la qualité de l'eau qui repose sur la comparaison des paramètres de qualité de l'eau avec les normes internationales ou nationales marocaines dans notre cas d'étude. En d'autres termes, l'IQE résume de grandes quantités de données sur la qualité de l'eau en termes simples (Excellente, Bonne, Mauvaise, Très mauvaise, etc.). Cette méthode a été initialement proposée par Horton (1965) et Brown et al. (1970). Dans cette étude l'indice IQE a été appliqué pour estimer l'influence des facteurs naturels et anthropiques sur la base de plusieurs paramètres clés du chimisme des eaux de surfaces de l'Oued Moulouya. Cet indice a été calculé en suivant la méthode de l'indice arithmétique pondéré (Brown et al., 1970 ; Brown et al., 1972 ; Chatterji et Raziuddin, 2002 ; Yidana et Yidana, 2010). Dans cette approche, une valeur numérique appelée poids relatif $\left(\mathrm{W}_{\mathrm{i}}\right)$, spécifique à chaque paramètre physico-chimique, est calculée (Tab.1) selon la formule suivante :

$$
\mathrm{W}_{\mathrm{i}}=\frac{\mathrm{k}}{\mathrm{S}_{\mathrm{i}}}
$$

Où :

$\mathrm{k}=$ constante de proportionnalité et peut également être calculée à l'aide de l'équation suivante :

$\mathrm{n}$ est le nombre de paramètres

$$
\mathrm{k}=\frac{1}{\sum_{\mathrm{i}=1}^{\mathrm{n}}\left(1 / \mathrm{S}_{\mathrm{i}}\right)}
$$

Si est la valeur maximale de la norme standard marocaine des eaux de surface (Norme Marocaine de qualité des eaux, 2002) de chaque paramètre en $\mathrm{mg} / \mathrm{l}$ sauf pour le $\mathrm{pH}$, la $\mathrm{T}^{\circ} \mathrm{C}$ et la conductivité électrique.

Ensuite, une échelle d'évaluation de la qualité (Qi) est calculée pour chaque paramètre en divisant la concentration par la norme dudit paramètre et en multipliant l'ensemble par 100 comme dans la formule suivante :

$$
\mathrm{Q}_{\mathrm{i}}=\left(\frac{\mathrm{C}_{\mathrm{i}}}{\mathrm{S}_{\mathrm{i}}}\right) \times 100
$$

Qi: échelle d'évaluation de la qualité de chaque paramètre.

$\mathrm{Ci}$ : la concentration de chaque paramètre en $\mathrm{mg} / \mathrm{l}$

Finalement l'indice global de la qualité de l'eau est calculé par l'équation suivante :

$$
\mathrm{IQE}=\sum_{\mathrm{i}=1}^{\mathrm{n}} \mathrm{Q}_{\mathrm{i}} \times \mathrm{W}_{\mathrm{i}} / \sum_{\mathrm{i}=1}^{\mathrm{n}} \mathrm{W}_{\mathrm{i}}
$$

Cinq classes de qualité peuvent être identifiées selon les valeurs de l'indice de qualité de l'eau IQE (Tab.1). 
Tableau 1. Classification et usage possible de l'eau selon l'IQE (Brown et al., 1972 ;

Chatterji et Raziuddin, 2002 ; Aher et al., 2016).

\begin{tabular}{|c|c|c|}
\hline Classe de IQE & Type d'eau & Usage possible \\
\hline $0-25$ & Excellente qualité & Eau potable, irrigation et industrie \\
\hline$>25-50$ & Bonne qualité & Eau potable, irrigation et industrie \\
\hline$>50-75$ & Mauvaise qualité & Irrigation et industrie \\
\hline$>75-100$ & Très mauvaise qualité & Irrigation \\
\hline$>100$ & Eau non potable & $\begin{array}{c}\text { Traitement approprié requis avant } \\
\text { utilisation }\end{array}$ \\
\hline
\end{tabular}

\section{Calcul de l'Indice de pollution organique (IPO)}

L'indice de pollution organique (IPO) de Leclercq (2001) a été également utilisé pour évaluer la charge organique dans la rivière. Dont le principe de l'IPO est de répartir les valeurs des éléments polluants en 05 classes (Tab.2). Cet indice s'obtient au moyen des valeurs des ammoniums, DBO5 et des phosphates. Le principe du calcul est de répartir les valeurs des trois éléments polluants en cinq classes et de déterminer, à partir des valeurs obtenues dans l'étude, le numéro de classe correspondant pour chaque paramètre en se servant des données moyennes du tableau 2. L'indice de pollution organique final est la moyenne des classes de pollution pour l'ensemble des paramètres (Tab.2).

Tableau 2. Grille des classes de l'indice de pollution organique (Leclercq, 2001).

\begin{tabular}{|c|c|c|c|c|c|}
\hline Classes & $\begin{array}{c}\mathbf{N H}_{4}{ }^{+} \\
(\mathbf{m g} / \mathbf{l})\end{array}$ & $\begin{array}{c}\text { DBO5 } \\
(\mathbf{m g O 2} / \mathbf{l})\end{array}$ & $\begin{array}{c}\mathbf{P O}_{4}{ }^{3-} \\
(\boldsymbol{\mu g} / \mathbf{l})\end{array}$ & IPO & $\begin{array}{c}\text { Pollution } \\
\text { organique }\end{array}$ \\
\hline 5 & $<0,1$ & $<2$ & $<15$ & $4,6-5,0$ & Nulle \\
\hline 4 & $0,1-0,9$ & $2,1-5$ & $16-75$ & $4,0-4,5$ & Faible \\
\hline 3 & $1-2,4$ & $5,1-10$ & $76-250$ & $3,0-3,9$ & Modérée \\
\hline 2 & $2,5-6$ & $10,1-15$ & $251-900$ & $2,0-2,9$ & Forte \\
\hline 1 & $>6$ & $>15$ & $>900$ & $1,0-1,9$ & Trés forte \\
\hline
\end{tabular}

\section{Résultat et discussion}

\section{Statistique descriptive}

Les caractéristiques statistiques descriptives des variables physicochimiques utilisées dans cette étude ont concerné les valeurs minimales et maximales, la moyenne et l'écart-type (Tab.3). Les résultats montrent que le $\mathrm{pH}$ est légèrement alcalin, ne présente pas une large différence entre les trois campagnes de prélèvement et varie entre 6,12 et 8,11 avec une valeur maximale observée durant la campagne mars-avril. La température varie entre 11,1 et $26,6^{\circ} \mathrm{C}$, cette dernière a été observée pendant la période juillet-août. La minéralisation des eaux est relativement élevée avec une conductivité électrique qui varie entre 413 et $2070 \mu \mathrm{S} / \mathrm{cm}$. La concentration de l'oxygène dissous OD diminue en fonction de la période de prélèvement, avec une valeur minimale enregistrée pendant juillet-août. En ce qui concerne les Nitrates et l'Ammonium, les résultats augmentent respectivement de 1,12 à 102,95 et de 
0,01 à 4,07mg/l. Les échantillons qui présentent les fortes concentrations en Ammonium et en Nitrates sont accompagnés par une forte charge polluante (39,55 mg/1 de $\mathrm{DBO}_{5}$ ). Les Sulfates oscillent entre 45et 279mg/l. Tous les paramètres montrent une augmentation légère mais nette depuis le mois de mars jusqu'au mois de juillet à part l'oxygène dissous qui voit ses teneurs à la baisse (Tab.3).

Tableau 3. Statistiques descriptives des paramètres physico-chimiques relatives aux trois campagnes de prélèvement de l'année 2014.

\begin{tabular}{|c|c|c|c|c|c|c|c|c|c|c|}
\hline & & pH & $\mathbf{T}$ & $\mathrm{CE}$ & OD & $\mathrm{NH}_{4}$ & $\mathrm{NO}_{3}$ & $\mathrm{SO}_{4}$ & $\mathrm{PO}_{4}$ & DBO5 \\
\hline & & & ${ }^{\circ} \mathbf{C}$ & $(\mu \mathrm{S} / \mathrm{cm})$ & $\mathrm{mg} / \mathrm{l}$ & $\mathrm{mg} / \mathrm{l}$ & $\mathrm{mg} / \mathrm{l}$ & mg/l & $\mathrm{mg} / \mathrm{l}$ & $\mathrm{mg} / \mathrm{l}$ \\
\hline \multirow[t]{4}{*}{$\begin{array}{l}\text { Base } \\
\text { totale }\end{array}$} & Min & 6,12 & 11,1 & 413 & 1,01 & 0,008 & 1,12 & 45 & 0,009 & 0,5 \\
\hline & Moyenne & 8,11 & 26,3 & 2070 & 9,8 & 4,07 & 102,95 & 279 & 2,79 & 39,55 \\
\hline & Max & 7,29 & 17,63 & 703,50 & 7,02 & 0,36 & 12,32 & 88,97 & 0,42 & 5,24 \\
\hline & $\begin{array}{c}\text { Écart- } \\
\text { type }\end{array}$ & 0,42 & 4,66 & 503,09 & 1,78 & 1,02 & 29,95 & 61,72 & 0,84 & 9,65 \\
\hline \multirow[t]{4}{*}{$\begin{array}{c}\text { Mars- } \\
\text { Avril }\end{array}$} & Min & 6,74 & 11,10 & 413,00 & 3,60 & 0,01 & 1,12 & 45,00 & 0,01 & 0,50 \\
\hline & Moyenne & 8,11 & 22,30 & 1972,00 & 9,80 & 2,65 & 97,61 & 199,00 & 1,95 & 25,55 \\
\hline & Max & 7,27 & 15,37 & 634,60 & 7,44 & 0,29 & 11,92 & 81,90 & 0,36 & 4,35 \\
\hline & $\begin{array}{l}\text { Ecart- } \\
\text { type }\end{array}$ & 0,42 & 4,16 & 477,07 & 1,67 & 0,83 & 30,12 & 52,88 & 0,73 & 7,61 \\
\hline \multirow[t]{4}{*}{ Mai-juin } & Min & 6,9 & 12,3 & 421 & 3,15 & 0,011 & 1,19 & 49 & 0,01 & 0,55 \\
\hline & Moyenne & 7,9 & 25,2 & 1970 & 9,11 & 3,26 & 101,21 & 225 & 2,54 & 33,15 \\
\hline & $\begin{array}{l}\text { Max } \\
\text { Écart- }\end{array}$ & 7,338 & 17,33 & 746,3 & 7,124 & 0,3509 & 12,38 & 86,4 & 0,4244 & 5,274 \\
\hline & & 0,39 & 4,55 & 578,95 & 1,59 & 1,02 & 31,22 & 60,65 & 0,89 & 9,95 \\
\hline \multirow[t]{4}{*}{$\begin{array}{c}\text { Juillet- } \\
\text { Août }\end{array}$} & 1VIIII & 6,12 & 15,1 & 453 & 1,01 & 0,011 & 1,27 & 53 & 0,011 & 1,05 \\
\hline & Moyenne & 7,8 & 26,3 & 2070 & 8,85 & 4,07 & 102,95 & 279 & 2,79 & 39,55 \\
\hline & $\begin{array}{l}\text { Max } \\
\text { Écart- }\end{array}$ & 7,255 & 20,18 & 729,6 & 6,508 & 0,4354 & 12,654 & 98,6 & 0,4653 & 6,102 \\
\hline & type & 0,50 & 4,36 & 494,66 & 2,10 & 1,28 & 31,74 & 75,05 & 0,97 & 11,88 \\
\hline
\end{tabular}

\section{Calcul des indices IQE et IPO et évaluation de la qualité des eaux} Indice de qualité de l'eau IQE

Dans cette étude, la qualité globale des eaux superficielles de la Haute Moulouya a été évaluée par la méthode de l'IQE. Le poids relatif $\left(\mathrm{W}_{\mathrm{i}}\right)$ de chaque paramètre physico-chimique et la constante de proportionnalité $\mathrm{k}$ ont été calculés en premier lieu en utilisant les valeurs maximales de la norme standard marocaine des eaux potables (NM 03.7.001, 2006) des paramètres physico-chimiques étudiés (Tab.4). En effet, 9 paramètres importants dans 
l'étude de la qualité des eaux de surface : $\mathrm{pH}, \mathrm{T}^{\circ} \mathrm{C}, \mathrm{CE}, \mathrm{OD}, \mathrm{NH}_{4}, \mathrm{NO}_{3}, \mathrm{SO}_{4}$, $\mathrm{PO}_{4}$ et $\mathrm{DBO}_{5}$ ont été pris en compte dans le calcul de la valeur de l'IQE.

Tableau 4. Poids des paramètres physico-chimiques et norme marocaine de qualité des eaux de surface (Norme Marocaine de qualité des eaux, NM 03.7.001, 2006).

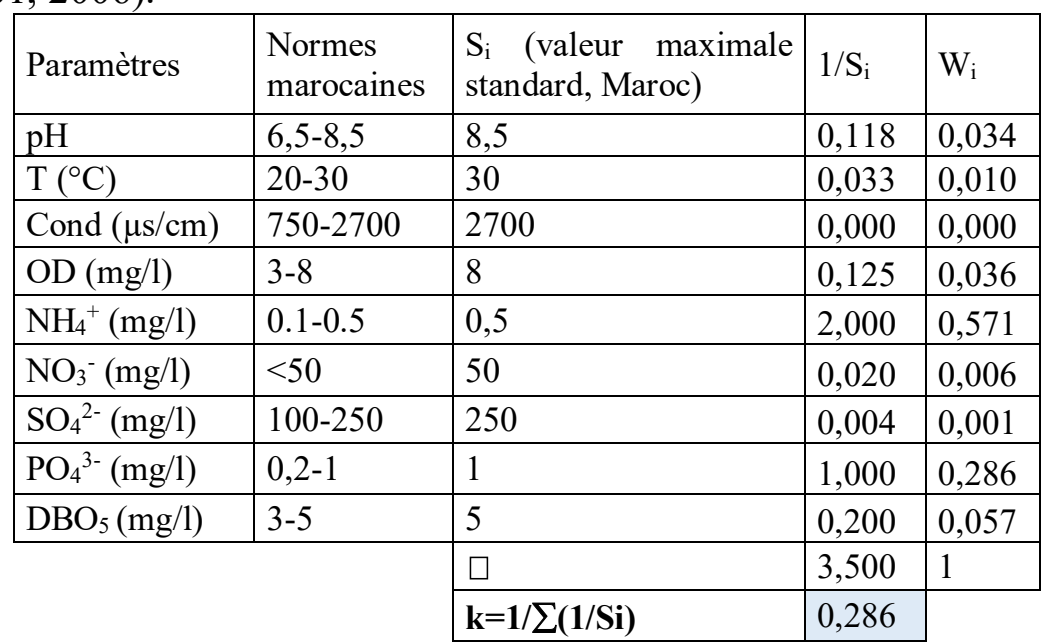

Après le calcul de l'indice global de qualité IQE en utilisant les résultats d'analyses physico-chimiques et les valeurs standards de la norme marocaine des eaux potables (NM 03.7.001, 2006), la classe de qualité des eaux est déterminée pour les 30 échantillons relatifs aux 10 stations d'échantillonnage (Tab.5). Ainsi, la classes de qualité excellente a été identifiée lors des campagnes mars-avril, mai-juin et juillet-aout pour les stations Ait Boulmane (M1), Ait Oha Ohaki (M2), Source Arbalou (M3), Krouchene (M4), Anzar Oufounas (M7), Aval Anzar Oufounas (M8), Anzegmir avant barrage (M9) et Anzegmir Amont (M10). Les classes de qualité mauvaise et non potable ont été enregistrées respectivement au niveau des stations de Boumia (M5) et de Zaida (M6) (Tab.5 et Fig.5). 
Tableau 5. Calcul de l'indice IQE et classe de qualité des eaux superficielles de la Haute Moulouya pendant les trois campagnes de l'année 2014.

\begin{tabular}{|c|c|c|c|c|c|c|}
\hline Stations & $\begin{array}{l}\text { IQE } \\
\text { Mars- } \\
\text { Avril } \\
\end{array}$ & $\begin{array}{l}\text { Classe de } \\
\text { qualité }\end{array}$ & $\begin{array}{l}\text { IQE } \\
\text { Mai- } \\
\text { juin }\end{array}$ & $\begin{array}{l}\text { Classe de } \\
\text { qualité }\end{array}$ & $\begin{array}{l}\text { IQE } \\
\text { Juillet- } \\
\text { Aout }\end{array}$ & $\begin{array}{l}\text { Classe } \\
\text { qualité }\end{array}$ \\
\hline M1 & 11.712 & Exce & 11,658 & nte & 11,929 & nte \\
\hline M2 & 11.502 & Excellente & 12,012 & Excellente & 12,261 & nte \\
\hline M3 & 11.441 & Excellente & 12,143 & Excellente & 12,257 & \\
\hline M4 & 17.063 & Excellente & 17,799 & Excellente & 18,690 & ente \\
\hline M5 & 62.254 & Mauvaise & 66,631 & Mauvaise & 71,344 & aise \\
\hline M6 & 393.923 & $\begin{array}{l}\text { Eau } \\
\text { potable }\end{array}$ & 489,140 & $\begin{array}{l}\text { Eau } \\
\text { potable }\end{array}$ & 594,997 & $\begin{array}{l}\text { Eau } \\
\text { potable }\end{array}$ \\
\hline M7 & 10.397 & Excellente & 10,361 & Excellente & 11,965 & Excellente \\
\hline M8 & 10.239 & Excellente & 10,489 & Excellente & 11,265 & Excellente \\
\hline M9 & 8.944 & & 9,109 & Ex & 9,602 & ente \\
\hline M10 & 10.814 & Excellente & 11,316 & Excellente & 11,969 & Excellente \\
\hline
\end{tabular}

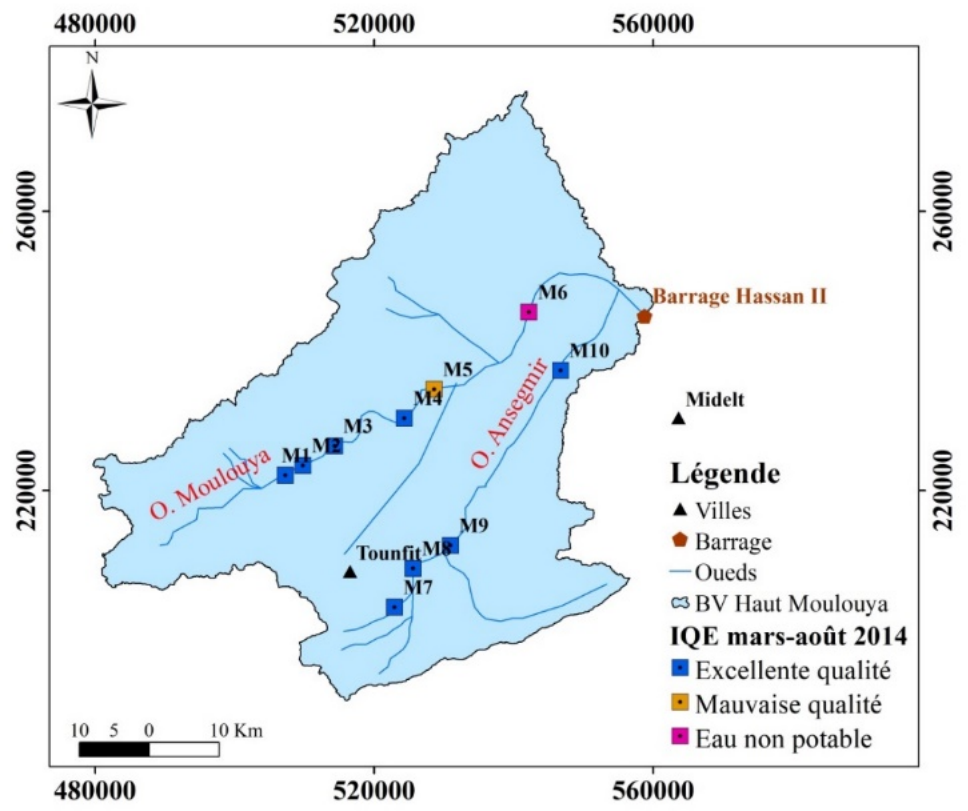

Figure 5. Carte thématique de l'indice de qualité globale IQE des eaux superficielles de la Haute Moulouya en amont du barrage Hassan pendant les campagnes mars-avril, mai-juin et juillet-août 2014.

\section{Indice de pollution organique IPO}

Après le calcul de l'indice de pollution organique IPO en utilisant les résultats d'analyses des paramètres indicateur de pollution $\left(\mathrm{NH}_{4}{ }^{+}, \mathrm{DBO}_{5}\right.$ et $\mathrm{PO}_{4}{ }^{3-}$ ), la classe de qualité des eaux est déterminée pour les trente échantillons relatifs aux dix stations d'échantillonnage (Tab.6 et Fig.6). Ainsi, cinq classes de pollution organique (pollution organique nulle, faible, modérée, forte et très 
forte) ont été identifiées lors des campagnes mars-avril, mai-juin et juillet-août 2014.

Le suivie d'analyse dans les différentes stations a fait l'objet d'un traitement de données par l'établissement des cartes thématiques de pollution organique des eaux superficielles de la Haute Moulouya durant les trois campagnes de prélèvement. Il renseigne sur la distribution spatio-temporelle de la qualité des eaux superficielles par le biais de l'indice de pollution organique IPO.

La classe de pollution organique nulle a été identifiée lors des trois campagnes mars-avril, mai-juin et juillet-aout pour les stations Ait Boulmane (M1), Ait Oha Ohaki (M2), Source Arbalou (M3), Krouchene (M4), Anzar Oufounas (M7), Aval Anzar Oufounas (M8), Anzegmir avant barrage (M9) et Anzegmir Amont (M10). Les classes de faible et forte pollution organique ont été observées respectivement au niveau des stations de Boumia (M5) et de Zaida (M6) (Tab.6 et Fig.6).

Tableau 6. Calcul de l'indice IPO des eaux superficielles de la Haute Moulouya pendant les trois campagnes de l'année 2014.

\begin{tabular}{|c|c|c|c|c|c|c|}
\hline Stations & $\begin{array}{c}\text { IPO } \\
\text { mars- } \\
\text { avril }\end{array}$ & $\begin{array}{c}\text { Pollution } \\
\text { organique }\end{array}$ & $\begin{array}{c}\text { IPO } \\
\text { mai-juin }\end{array}$ & $\begin{array}{c}\text { Pollution } \\
\text { organique }\end{array}$ & $\begin{array}{c}\text { IPO } \\
\text { juillet- } \\
\text { août }\end{array}$ & $\begin{array}{c}\text { Pollution } \\
\text { organique }\end{array}$ \\
\hline M1 & 5,0 & nulle & 5,0 & nulle & 5,0 & nulle \\
\hline M2 & 5,0 & nulle & 5,0 & nulle & 5,0 & nulle \\
\hline M3 & 5,0 & nulle & 5,0 & nulle & 4,7 & nulle \\
\hline M4 & 4,7 & nulle & 4,7 & nulle & 4,7 & nulle \\
\hline M5 & 4,3 & faible & 4,3 & faible & 4,3 & faible \\
\hline M6 & 2,7 & forte & 2,7 & forte & 2,7 & forte \\
\hline M7 & 5,0 & nulle & 5,0 & nulle & 5,0 & nulle \\
\hline M8 & 5,0 & nulle & 5,0 & nulle & 5,0 & nulle \\
\hline M9 & 5,0 & nulle & 5,0 & nulle & 5,0 & nulle \\
\hline M10 & 4,7 & nulle & 4,7 & nulle & 4,7 & nulle \\
\hline
\end{tabular}




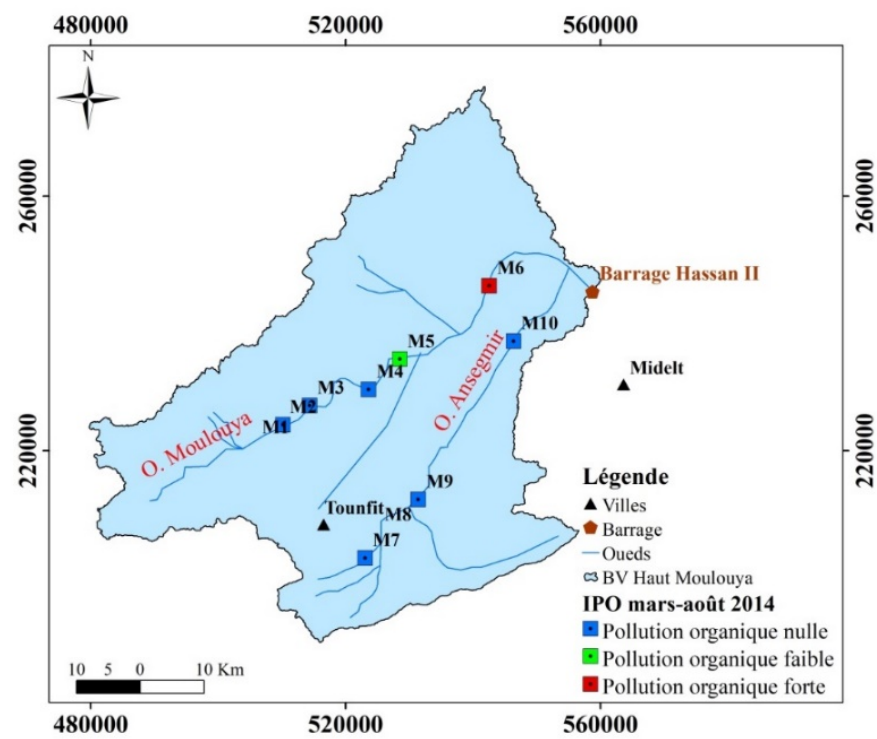

Figure 6. Carte thématique de l'indice de pollution organique IPO des eaux superficielles de la Haute Moulouya en amont du barrage Hassan pendant les campagnes mars-avril, mai-juin et juillet-août 2014.

\section{Discussion}

L'indice de qualité de l'eau IQE pendant les trois compagnes mars-avril, mai-juin et juillet-août de l'année 2014 indique que la majorité des stations (M1, M2, M3, M4, M7, M8, M9 et M10), soit 80\% des stations, restent d'excellente qualité $(0<\mathrm{IQE} \leq 25)$. Les deux stations restantes, représentant $10 \%$ chacune de l'ensemble des stations, demeurent également sans changement saisonnier avec une mauvaise qualité $(50<\mathrm{IQE} \leq 75)$ pour la station M5 de la commune de Boumia, et des eaux non potables (IQE> 100) pour la station M6 de la commune de Zaida, Cependant, la classe de bonne qualité reste absente (Tab.5 et Fig.5). Le degré croissant de la dégradation des eaux entre les stations M5 et M6 est lié à l'augmentation de l'impact démographique et urbanistique de la commune de Zaida par rapport à celui de Boumia. Des résultats concordants et très similaires ont été aussi observés dans la zone d'étude sur la base de l'indice IQE (Talhaoui et al., 2020) et de l'indice de pollution IP (El Hmaidi et al., 2020).

L'état de pollution des eaux superficielles de la Haute Moulouya, calculé à partir de l'indice de pollution organique IPO, montrent que $80 \%$ des stations ( 8 stations) ne présentent aucune pollution organique $(4,5 \leq \mathrm{IPO} \leq 5)$ durant les trois campagnes de 2014 (mars-avril, mai-juin et juillet-août). L'exception apparait une autre fois au niveau de la station de Boumia (M5), représentant $10 \%$ des stations, qui indique une pollution organique faible $(4 \leq \mathrm{IPO} \leq 4,5)$ et de la station de Zaida (M6) qui affiche des eaux avec de fortes pollutions organiques $(2 \leq \mathrm{IPO} \leq 3)$ (Tab.6 et Fig.6). Cette pollution est surtout liée aux 
concentrations relativement élevées en $\mathrm{DBO}_{5}$ qui dépassent les normes marocaines de potabilité $5 \mathrm{mg} / 1$ (Taybi et al., 2016). La dégradation de la qualité des eaux au niveau des stations M5 et M6 respectivement des communes de Boumia et de Zaida le long de l'oued Moulouya est due aux activités agricoles et aux rejets des eaux usées sans aucun traitement préalable (Serge et Ernest, 2020).

Les résultats des différents indices, à savoir l'indices de qualité de l'eau (IQE) et l'indice de pollution organique (IPO), appliqués aux eaux superficielles de la Haute Moulouya étudiées durant les trois campagnes de 2014 (mars-avril, mai-juin, juillet-août), ont été comparés entre eux afin de tester le degré de concordance et / ou de discordance entre les différentes méthodes. Les deux indices IQE et IPO montrent une complète concordance entre toutes les stations et durant les trois campagnes. La comparaison avec les travaux antérieurs montre aussi une bonne concordance avec les indices globaux de qualité IQE et de pollution IP (Talhaoui et al., 2020 ; El Hmaidi et al., 2020).

De loin, la qualité et le degré de pollution organique des eaux étudiées paraissent stables sans changement apparent. Cependant, l'examen détaillé des résultats met en évidence une nette variation saisonnière avec une légère tendance à la dégradation. Ainsi, les valeurs minimales, moyennes et maximales de l'indice IQE augmentent légèrement en allant de la saison humide mars-avril à mai-juin vers la saison sèche de juillet-août de l'année 2014 (Tab.7). Cette variation saisonnière a été largement signalée dans la littérature aussi bien dans la zone d'étude (Taybi et al., 2016 ; Talhaoui et al., 2020 ; El Hmaidi et al., 2020) qu'ailleurs (Vital et al., 2018 ; Serge et Ernest, 2020 ; Miriac et al., 2020).

Tableau 7. Statistiques descriptives de l'indice IQE et IPO pendant les trois campagnes de l'année 2014.

\begin{tabular}{|c|c|c|c|c|c|c|}
\cline { 2 - 7 } \multicolumn{1}{c|}{} & \multicolumn{3}{c|}{ IQE } & \multicolumn{3}{c|}{ IPO } \\
\cline { 2 - 7 } \multicolumn{1}{c|}{} & Mars-avril & Mai-juin & Juillet-août & Mars-avril & Mai-juin & Juillet-août \\
\hline Min & 8,94 & 9,11 & 9,60 & 2,67 & 2,67 & 2,67 \\
\hline Max & 393,92 & 489,14 & 595,00 & 5,00 & 5,00 & 5,00 \\
\hline Moyenne & 54,83 & 71,00 & 76,63 & 4,63 & 4,63 & 4,60 \\
\hline Écart-type & 120,23 & 150,01 & 183,09 & 0,73 & 0,73 & 0,72 \\
\hline
\end{tabular}

La détérioration de la qualité des eaux est traduite par une importante charge minérale et organique d'origine anthropique en provenance des communes urbaines de Boumia et Zaida dans la Haute Moulouya. Ces eaux sont très polluées au niveau de ces communes, à causes des concentrations très élevées des nitrates, de l'ammonium et de $\mathrm{DBO}_{5}$ qui dépassent les normes marocaines de potabilité de 0,5 pour l'Ammonium, $50 \mathrm{mg} / 1$ pour les Nitrates et $5 \mathrm{mg} / 1$ pour la $\mathrm{DBO}_{5}$. Cette dégradation est principalement d'origine anthropique en relation avec les activités agricoles par lessivage des sols très 
chargés en fertilisants et avec les rejets des eaux usées urbaines (Taybi et al., 2016; Charles Vital et al., 2018). Des travaux similaires ont montré que l'augmentation du degré de pollution en été serait liée, sans doute, à la diminution des débits de l'oued Moulouya alors que ceux des effluents chargés en eaux usées domestiques et industrielles provenant des différents centres urbains (Boumia et Zaida) restent importants (Talhaoui et al., 2020 ; El Hmaidi et al., 2020).

\section{Conclusion}

La qualité des eaux des rivières, barrages et nappes phréatiques s'est fortement dégradée, limitant ainsi le potentiel réel en eau et aboutissant à des impacts sanitaires et écologiques importants. Les effluents urbains et l'utilisation des produits chimiques agricoles et industriels, rejetés trop souvent sans traitements appropriés dans le milieu récepteur, constituent la source principale de la dégradation de la qualité des eaux superficielles. L'agriculture participe à cette dégradation par l'utilisation exagérée de fertilisants azotés et phosphatés, pesticides, herbicides, etc., d'où le phénomène de la pollution des eaux.

Le suivi des indices de qualité (IQE) et de pollution organique (IPO) des eaux au cours de cette étude montre que les eaux superficielles de la Haute Moulouya restent à $80 \%$ d'excellente qualité et sans pollution organique (IPO). La variation saisonnière n'apparaît que lors de l'examen fin et détaillé de l'IQE et traduit une légère tendance à la dégradation des eaux de la Haute Moulouya entre les hautes eaux (mars-avril, mai-juin) et les basses eaux (juillet-août) de l'année 2014. Toutefois, les stations urbaines affichent une détérioration croissante de la qualité de leurs eaux en fonction de la pression démographique des communes. Ainsi les eaux superficielles sont de mauvaise qualité avec une pollution organique faible dans la commune de Boumia et deviennent de plus en plus dégradées au niveau de la commune urbaine de Zaida avec des eaux non potables et à forte charge de pollution organique. Cette pollution anthropique en aval des communes urbaines est due aux teneurs très élevées en certains paramètres physico-chimiques (Nitrates, Ammonium et $\mathrm{DBO}_{5}$ ) dépassants les normes et sont en relation directe avec les rejets des eaux usées sans traitement provenant des communes de Boumia et Zaida et en degré moindre avec les fertilisants azotés et phosphatés utilisés en agricultures.

\section{References :}

1. ABHM (Agence du bassin hydraulique de la Moulouya), (2009). Etude du plan directeur d'aménagement intégré des ressources en eau du bassin de la Moulouya (PDAIRE), Mission II, Développement des ressources en eau du Bassin, 185p. 
2. Ahamrouni, J. (1996). Erosion hydrique dans le bassin versant de la Moulouya (Maroc Oriental): Recherche des zones sources d'envasement de la retenue du barrage Mohamed V. Thèse de $3^{\text {ème }}$ cycle, Université Cheikh Anta Diop, Faculté des Sciences et Techniques, Dakar, Sénégal, $147 \mathrm{p}$.

3. Aher, D. N., Kele, V. D., Malwade, K. D., \& Shelke, M. D. (2016). Lake Water Quality Indexing To Identify Suitable Sites For Household Utility: A Case Study Jambhulwadi Lake; Pune (MS). Int. Journal of Engineering Research and Applications, 6(5), (pp.16-21).

4. Amrani, A. (2007). Apport des données couplées d'imagerie satellitaire Etm+ de landsat 7 et modèle numérique de terrain appliques à l'étude morphogénétique de la haute et moyenne Moulouya (Maroc). Thèse Faculté des Sciences Agdal de Rabat - Doctorat ou Doctorat National [559] ,217p.

5. Azzaoui S., EL Hanbali M., Leblanc M. (2002). Copper, lead, iron and manganese in the Sebou drainage basin; sources and impact on surface water quality. Water Quality Research Journal Canada 37(4), 773-784.

6. Brown R.M., McClelland N.I., Deininger R.A., O'Connor M.F. (1972). A Water Quality Index - Crashing the Psychological Barrier. In: Thomas W.A. (eds) Indicators of Environmental Quality. Environmental Science Research, vol 1, 173-182. Springer, Boston, MA. https://doi.org/10.1007/978-1-4684-2856-8_15

7. Brown, R. M., McClelland, N. I., Deininger, R. A., Tozer, R. G. (1970). A Water Quality Index- Do We Dare? Water and Sewage Works, 117, 339-343.

8. Chahboune M., Chahlaoui A. and Zaid A., (2014). Contribution to comparative study of the physico-chemical quality of waters of Moulouya and Ansegmir rivers in upstream of Hassan II dam (Province of Midelt, Morocco). Journal of Biodiversity and Environmental Sciences (JBES), 4(2), 278-288. ISSN: 2220-6663 (Print) 2222-3045 (Online).

9. Chatterji, C., Raziuddin, M. (2002). Determination of water quality index of a degraded river in Asanol Industrial area, Raniganj, Burdwan, West Bengal. Nature, Environment and Pollution Technology, 1 (2) (pp. 181-189).

10. Combe, M. \&, Simonot, M. (1971). La Haute Moulouya, le sillon d'Itzer-Enjil et le massif de Bou-Mia Aouli. Ressources en eau du Maroc (Tome1). Domaine du Rif et du Maroc oriental. Notes et Mém. Serv. Géol., Maroc : 193-201.

11. Defranceschi M. (1996). L'eau dans tous ses états. Éditions Ellipses, Paris, 123 pp. 
12. Diani K., Tabyaoui H., Kacimi I., El Hammichi F., Nakhcha Ch., (2017). Stream Network Modelling from Aster GDEM Using ArcHydro GIS: Application to the Upper Moulouya River Basin (Eastern, Morocco). Journal of Geoscience and Environment Protection, 5, 1-13. https://doi.org/10.4236/gep.2017.55001

13. El Hachimi, M. L., El Founti, L., Bouabdli, A., Saidi, N., Fekhaoui, M., \& Tassé, N. (2005). Impact de la mine abandonnée de Zeïda (Maroc) sur la qualité des eaux de surface. Bulletin de la Société d'Histoire Naturelle de Toulouse, 141-1, 81-88.

14. El Hmaidi A., Talhaoui A., Manssouri I., Jaddi H., Ousmana H., 2020. Contribution of the pollution index and GIS in the assessment of the physico-chemical quality of the surface waters of Moulouya River (NE, Morocco). La Houille Blanche, 3, 45-54. https://doi.org/10.1051/lhb/2020028

15. Horton, R. K. (1965). An index number system for rating water quality. Journal of Water Pollution Control Federation, 37(3), (pp.300306).

16. Khamar M., Bouya D., Ronneau C. (2000). Pollution métallique et organique des eaux et des sédiments d'un cours d'eau marocain par les rejets liquides urbains. Water Quality Research Journal, Canada 35 (1), 147- 161.

17. Laabidi, A., Gourari, L., El Hmaidi, A., Aarab, M. \& Gretaa, M., 2014. Caractérisation géomorphologique, lithostratigraphique et sédimentologique des dépôts quaternaires de l'Oued Bou Salloum (Haute Moulouya, Maroc). International Journal of Engineering Research and Development, 10 (4), 7-19. e-ISSN: 2278-067X, pISSN: 2278-800X.

18. Lalami, A. E. O., Merzouki, M., El Hillali, O., Maniar, S., \& Koraichi, S. I. (2011). Pollution des eaux de surface de la ville de Fès au Maroc: typologie, origine et conséquences. LARHYSS Journal P-ISSN 11123680/E-ISSN 2521-9782, (9).

19. Leclercq L. (2001). Les eaux courantes : caractéristiques et moyens d'étude, dans les zones humides. Actes des colloques organisés en 1996 par le Ministère de la Région Wallonne dans le cadre de l'Année Mondiale des Zones Humides, Jambes, Région Wallonne, DGRNE. pp. 67-82.

20. Merabet S. (2010). Évaluation de la qualité physico-chimique des eaux brutes et distribuées du barrage réservoir de Beni Haroun. Mémoire de magister chimie analytique. Université Mentouri de Constantine, Algérie, 104p.

21. Miriac, A. S. D., Noukpo, A., \& Christophe, H. S. (2020). Eau De Consommation Et Maladies Hydriques Dans La Commune De 
Lokossa $\mathrm{Au}$ Sud-Ouest De La Republique Du Benin (Afrique De L'ouest). European Scientific Journal, ESJ, 16(15), 393-4017. https://doi.org/10.19044/esj.2020.v16n15p393.

22. Mutin G. (2000). L'eau dans le monde arabe. Carrefours de géographie, 184 p., 25 cartes et figures. Paris, Edition Ellipse.

23. Myers D.N., (2015). Foundations of Water Quality Monitoring and Assessment in the United States. Elsevier Inc. p.21-92.

24. Naji, M. (2004). Les minéralisations plombo-barytiques du district de la Haute Moulouya : Contexte géologique, contrôle tectonique et modèle de mise en place - Gisements d'Aouli-Mibladène, Maroc. Thèse en géologie appliquée, Université Mohammed V, Rabat, $218 \mathrm{p}$.

25. Nasloubi, M. (1993). Genèse et modélisation des minéralisations plombifère de la haute Moulouya (Maroc). Mémoire de maitrise, Université du Québec à Montréal, 107p.

26. NM 03.7.001., 2006. Norme marocaine relative à la qualité des eaux d'alimentation humaine. Rabat, Bulletin Officiel $N^{\circ} 5404$.

27. Normatov P. I., Armstrong R., Normatov I. S., Narzulloev N., Russ. Meteorol. (2015). Hydro+. 40 p 347-354.

28. Observatoire régional de la Sante Rhone-Alpes, (2007). Les nitrates, Tableau de bord santé-environnement, région Rhône-Alpes, $16 \mathrm{p}$.

29. Rodier J., 2009. L'analyse de l'eau, $9^{\mathrm{e}}$ édition. DUNOD (éditeur), Paris, France. 1579 p.

30. Serge, Y. K., \& Ernest, A. K. (2020). Caractérisation PhysicoChimique Des Eaux De Surface Dans Un Environnement Minier Du Centre-Ouest De La Côte d'Ivoire : Cas Du Département De Divo. European Scientific Journal, ESJ, 16(12), 293-315. https://doi.org/10.19044/esj.2020.v16n12p293.

31. Talhaoui A, El Hmaidi A, Jaddi H, Ousmana H, Manssouri I. (2020). Calcul de L'Indice de Qualité de l'Eau (IQE) pour l'évaluation de la qualité physico-chimique des eaux superficielles de L'Oued Moulouya (NE, Maroc). European Scientific Journal, ESJ, 16(2): 64-85. https://doi.org/10.19044/esj.2020.v16n2p64.

32. Taybi, A.F., Mabrouki, Y., Berrahou, A., Chaabane, K. (2016). Évolution spatiotemporelle des paramètres physico-chimiques de la Moulouya. Journal of Materials and Environmental Science, 7 (1) (pp. 272-284).

33. Vital, S. N. C., Robert, N., \& Benoît, N. M. (2018). Pollution De L'eau De Consommation Humaine Et Risques Sanitaires A Court Terme : Cas Du Bassin Versant De La Menoua (Ouest-Cameroun). European Scientific Journal, ESJ, 14(3), 96-117. https://doi.org/10.19044/esj.2018.v14n3p96. 
34. Yidana, S. M. \& Yidana, A. (2010). Assessing water quality using water quality index and multivariate analysis. Environmental Earth Sciences, 59(7) (pp.1461-1473). https://doi.org/10.1007/s12665-0090132-3. 